\title{
Comparison of cervical cancer screening among women with and without hysterectomies: a nationwide population- based study in Korea
}

\author{
Ji-Yeon Shin ${ }^{1}$, Kui Son Choi ${ }^{2,3}$, Mina Suh ${ }^{3}$, Boyoung Park ${ }^{3,4}$ and Jae Kwan Jun ${ }^{2,3^{*}}$ (1)
}

\begin{abstract}
Background: Cervical cancer screening is not recommended for women who underwent hysterectomy with no history of cervical intraepithelial neoplasia $(\mathrm{CIN})$ of grade 2 or higher. We aimed to determine the cervical cancer screening rate in Korean women who underwent hysterectomies and compare it to that in women with intact uteri.

Methods: We used data from the 2014-2016 Korean National Cancer Screening Survey; 6807 women aged 3074 years were included in the study. Participants were asked about their experiences with cervical cancer screening, hysterectomy status, and other variables associated with cancer screening.

Results: The screening rates among women who have undergone a hysterectomy vs. those who have not during the past 2 years were $61.8 \%$ (95\% confidence interval [Cl], 58.8-64.9) and $64.7 \%(95 \% \mathrm{Cl}, 64.1-65.3)$, respectively. Among younger women (30-44 years) and women with a family history of cancer, those with hysterectomies showed a higher cervical cancer screening rate than those without ( $77.8 \%$ vs. $57.1 \%$ and $75.0 \%$ vs. $67.1 \%$, respectively).

Conclusions: Despite available evidence and clinical recommendations, a considerable number of Korean women who no longer have a cervix continue to undergo unnecessary cervical cancer screening. It is necessary to identify the exact underlying causes for this phenomenon, and systematic efforts are required to prevent unnecessary screening for women who have undergone a hysterectomy.
\end{abstract}

Keywords: Uterine cervical neoplasms, Mass screening, Hysterectomy, Papanicolaou test, Guideline

\section{Background}

Cervical cancer is one of the most common cancers among women worldwide. Approximately 530,000 new cases of cervical cancer were diagnosed in 2012, and 270,000 women died of the disease [1].Therefore, several countries have made efforts to reduce the burden of this cancer, and one such endeavor is promoting cervical cancer screening programs $[2,3]$. Since the introduction of cervical cancer screening in the 1950s and 1960s, the incidence and mortality rates due to cervical cancer have

\footnotetext{
* Correspondence: jkjun@ncc.re.kr

${ }^{2}$ Graduate School of Cancer Science and Policy, National Cancer Center, 323

Ilsan-ro, Ilsandong-gu, Goyang 10408, Republic of Korea

${ }^{3}$ National Cancer Control Institute, National Cancer Center, 323 IIsan-ro,

Ilsandong-gu, Goyang 10408, Republic of Korea

Full list of author information is available at the end of the article
}

dramatically decreased in western countries [4-6]. In Korea, screening programs were formulated by the Korean government in 1996; the government formally established the National Cancer Screening Program (NCSP) for cervical cancer in 1999. The NCSP recommends that women aged $\geq 30$ years undergo cervical cancer screening on a biennial basis via the Papanicolaou (Pap) smear [7]. Since then, the age-standardized incidence rate of cervical cancer has been decreasing steadily from 16.3 per 100,000 in 1995 to 9.0 per 100,000 in 2014 [8].

Hysterectomy is the most common non-pregnancy related surgical procedure for women of reproductive age $[9,10]$. More than one-third of all women in the United States have had a hysterectomy by the age of 60 years [11]. In Korea, hysterectomies were the sixth most

(c) The Author(s). 2018 Open Access This article is distributed under the terms of the Creative Commons Attribution 4.0 International License (http://creativecommons.org/licenses/by/4.0/), which permits unrestricted use, distribution, and reproduction in any medium, provided you give appropriate credit to the original author(s) and the source, provide a link to the Creative Commons license, and indicate if changes were made. The Creative Commons Public Domain Dedication waiver (http://creativecommons.org/publicdomain/zero/1.0/) applies to the data made available in this article, unless otherwise stated. 
common type of surgery in 2015 [12]. According to recent consensus guidelines, cervical cancer screening is not recommended for women who undergo total hysterectomy for benign diseases [6, 13-15]. In 1996, the United States Preventive Services Task Force (USPSTF) first recommended that women discontinue screening if they have undergone a total hysterectomy and had no history of cervical cancer $[6,13]$. In 2012, the American Cancer Society and the American College of Obstetricians and Gynecologists recommended that women of all ages should forgo screening for vaginal cancer using any modality following a hysterectomy that included removal of the cervix if they have no history of cervical intraepithelial neoplasia (CIN) of grade 2 or higher [15]. In Korea, the National Cervical Cancer Screening Guideline Development Committee stated in 2015 that cervical cancer screening is not recommended for women who underwent a total hysterectomy [16].

Despite the 1996 USPSTF recommendations, the 2010 National Health Interview Survey found that $55.8 \%$ of women in the United States who had hysterectomies still reported having undergone cervical cancer screening in the prior three years [17]; the USPSTF also pointed out the unnecessary use of healthcare resources and ensuing costs resulting from this phenomenon. However, no studies have examined the actual status of cervical cancer screening for women who received hysterectomies in Korea. In this study, we aimed 1) to review and summarize the cervical cancer screening recommendations for women who underwent total hysterectomies and 2) to determine and compare the rate of cervical cancer screening among women with and without hysterectomies in Korea.

\section{Methods}

\section{Study design and population}

We used data from the Korean National Cancer Screening Survey (KNCSS) performed between 2014 and 2016. The KNCSS is a continuous nationwide cross-sectional interview survey conducted by the Korean National Cancer Center annually since 2004 to examine the participation rates of Koreans in screening for five common cancers: gastric, liver, colorectal, breast, and cervical. The details of the surveying methods were described previously [7]. Briefly, survey participants were selected based on the Resident Registration Population data using a stratified, multistage, random sampling procedure according to residential area, age, and sex. Investigators from a professional research agency conducted face-to-face interviews in the participants' homes. They obtained informed consent from all participants. Eligible participants were asked about their experiences related to screening for the five aforementioned cancers and provided information on health behaviors, health status, family history of cancer, and sociodemographic factors.

Surveys were completed by 4500 participants aged 20 years or older in 2014, 4500 in 2015, and 4500 in 2016; the response rates were $40.9,66.0$ and $48.1 \%$, respectively. We selected 6807 cancer-free women aged $30-74$ years as the study population, as women aged $\geq 30$ years are eligible to undergo cervical cancer screening biennially via Pap smear, according to the NCSP protocols [7], and cervical cancer screening is recommended to be terminated at the age of 74 years, after three consecutive negative cervical cancer screening results [16]. Since 2005, the NCSP has provided Medical Aid recipients and NHI beneficiaries in the lower $50 \%$ income stratum with cervical cancer screening free of charge. NHI beneficiaries in the upper $50 \%$ income stratum receive cervical cancer screening services from the NHI Corporation and pay $10 \%$ of the screening cost [7]. All participants provided answers to the relevant questions regarding experience or timing of cervical cancer screening as well as undergoing hysterectomy and any Pap smears beforehand.

\section{Measures}

Using a structured self-reported questionnaire (Additional file 1), participants were probed regarding their experiences with cervical cancer screening and hysterectomy status. In terms of hysterectomy, participants were asked: "Have you received a hysterectomy?" and "When did you received a hysterectomy?" Regarding cervical cancer screening, participants were asked "Have you ever undergone cervical cancer screening?" and "When was the last time you had a cervical cancer examination?" The "cervical cancer screening rate with recommendation' was defined as the proportion of participants who underwent a Pap smear within the previous two years according to NCSP recommendations.

As in previous studies investigating factors associated with cancer screening $[18,19]$, we considered age group (30-44, 45-64, and 65-74 years), residential area (metropolitan, urban, or rural), education $(\leq 11$ or $\geq$ 12 years of schooling), family history of cancer (yes or no), health insurance status (medical aid program [MAP] or the National Health Insurance [NHI]), possession of private health insurance (yes or no), and monthly household income group $(<2500,2500-4499$, or $\geq 4500$ USD) as covariates.

\section{Statistical analysis}

We used the chi-square test to investigate the relationships between sociodemographic characteristics among women with vs. without hysterectomies. Univariable and multivariable logistic regression models were used to compare the cervical cancer screening rates between 
these two groups within each subgroup of covariates. In the multivariable analysis, all covariates except the subgroup variable were included in the model, and the adjusted odds ratios (aORs) and 95\% confidence intervals (CIs) were calculated. For example, in order to calculate the aOR of undergoing cervical cancer screening in ages $30-44$ years, a logistic regression analysis was performed for ages 3044 years that included hysterectomy status (reference group: reporting no hysterectomy), and other covariates, except for age-groups, as independent variables. All statistical analyses were performed using the SAS software package (version 9.4, SAS Institute, Cary, NC, USA), and appropriate sampling weights were included with a survey procedure to reflect the complex survey design. Sampling weight is a measure of the number of people in the population and represents a sample of the KNCSS and reflects the unequal probability of selection, nonresponse adjustment, and adjustment to independent population controls. The use of sample weights allows us to obtain an unbiased national estimate. This study was approved by the Institutional Review Board of the National Cancer Center, Korea (approval no. NCCNCS-08-129).

\section{Results}

Table 1 summarizes the cervical cancer screening guidelines for women who have undergone a hysterectomy. In 1996, the USPSTF first recommended against cervical cancer screening for women who have undergone a total

Table 1 Cervical cancer screening guidelines for women who have undergone a hysterectomy

\begin{tabular}{lclc}
\hline Guideline & Year & Recommendation & Ref. \\
\hline ASCCP/ & 2012 & Women at any age following a hysterectomy & {$[15]$} \\
ACS/ASCP & & with removal of the cervix who have no \\
& history of CIN2+ should not be screened for \\
& vaginal cancer using any modality.
\end{tabular}

2012 There is convincing evidence that continued screening after hysterectomy with removal of the cervix for indications other than a highgrade precancerous lesion or cervical cancer provides no benefits.

NCCN 2010 Women who have had a total hysterectomy for benign indications and have no prior history of high-grade CIN should discontinue routine cytology testing.

KSGO 2012 Women who have undergone hysterectomy should continue with screening tests if they have a history of CIN grade 2 or higher or if they do not know their pervious cytology results.

NCSP

2015 Cervical cancer screening was not recommended for women who underwent hysterectomy and women with no history of CIN grade 2 or higher.

ASCCP American Society for Colposcopy and Cervical Pathology, ACS American Cancer Society, ASCP American Society for Clinical Pathology, USPSTF U.S Preventive Services Task Force, NCCN National Comprehensive Cancer Network, KSGO Korean Society of Gynecologic Oncology, NCSP National Cancer Screening Program, CIN Cervical Intraepithelial Neoplasia hysterectomy for benign disease [13]; two subsequent cervical cancer screening guideline updates by the USPSTF in 2003 and 2012 [6, 20] maintained the same guidelines. The USPSTF recommended against screening for cervical cancer in women who have had a hysterectomy with removal of the cervix and who do not have a history of a high-grade precancerous lesion (CIN grade 2 or 3) or cervical cancer (D recommendation) in 2012 [6]. In Korea, the National Cervical Cancer Screening Guideline Development Committee published in 2015 stated that cervical cancer screening is not recommended for women who underwent total hysterectomy and had no history of CIN grade 2 or higher lesions [16].

Of the 6807 survey respondents, 249 (3.7\%) reported having undergone a hysterectomy. Table 2 shows the general characteristics of women who underwent a hysterectomy vs. those who did not. Women who underwent hysterectomies were older, less educated, and had lower incomes than women who did not undergo hysterectomies. The proportions of women who underwent Pap smears in the previous year were $30.9 \%$ in the hysterectomy group and $30.3 \%$ in the non-hysterectomy group, indicating similar proportions.

The screening rates among women who underwent a hysterectomy during the previous two years vs. those who did not were $61.8 \%$ (95\% CI, 58.8-64.9) and 64.7\% (95\% CI, 64.1-65.3), respectively (Table 3). In the hysterectomy group, a younger age and higher income level coincided with a higher screening rate. Among participants who were 30-44 years of age or participants with a family history of cancer, those who underwent hysterectomies had higher screening rates than those who did not $(77.8 \%$ vs. $57.1 \%$ and $75.0 \%$ vs. $67.1 \%$, respectively).

Table 3 shows the results of multivariable analyses performed to identify factors associated with cervical cancer screening in the hysterectomy group vs. the non-hysterectomy group. Women who underwent hysterectomies in the following categories were less likely to undergo cervical cancer screening than their counterparts with intact uteri: Metropolitan residents, those with longer education, those with no family history of cancer, those who used the NHI, those with private health insurance, and those with monthly household incomes group $<2500$ USD. Even after controlling for the other variables, subjects in the 30-44-year age group and those with a family history of cancer were more likely to undergo cervical cancer screening than women who had not undergone hysterectomies, although the differences were not were significant.

\section{Discussion}

We found that $61.8 \%$ of women with hysterectomies underwent cervical cancer screening within the previous 
Table $\mathbf{2}$ General characteristics of the study population

\begin{tabular}{|c|c|c|c|c|c|}
\hline \multirow[t]{2}{*}{ Characteristics } & \multicolumn{2}{|c|}{ Reporting no hysterectomy } & \multicolumn{2}{|c|}{ Reporting hysterectomy } & \multirow{2}{*}{$\begin{array}{l}p- \\
\text { value }\end{array}$} \\
\hline & $n$ & Weighted \% & $n$ & Weighted \% & \\
\hline \multicolumn{6}{|l|}{ Age, years } \\
\hline $30-44$ & 2436 & 37.2 & 18 & 7.2 & \multirow[t]{3}{*}{$<0.001$} \\
\hline $45-64$ & 3392 & 51.7 & 167 & 67.1 & \\
\hline $65-74$ & 730 & 11.1 & 64 & 25.7 & \\
\hline \multicolumn{6}{|l|}{ Residence area } \\
\hline Metropolitan & 3003 & 45.8 & 122 & 49.0 & \multirow[t]{3}{*}{0.335} \\
\hline Urban & 3036 & 46.3 & 113 & 45.4 & \\
\hline Rural & 519 & 7.9 & 14 & 5.6 & \\
\hline \multicolumn{6}{|l|}{ Education, years } \\
\hline$\leq 11$ & 897 & 13.7 & 68 & 27.3 & \multirow[t]{2}{*}{$<0.001$} \\
\hline$\geq 12$ & 5661 & 86.3 & 181 & 72.7 & \\
\hline \multicolumn{6}{|c|}{ Family history of cancer } \\
\hline Yes & 887 & 13.5 & 80 & 32.1 & \multirow[t]{2}{*}{$<0.001$} \\
\hline No & 5671 & 86.5 & 169 & 67.9 & \\
\hline \multicolumn{6}{|c|}{ Health insurances status } \\
\hline $\mathrm{NHI}$ & 6480 & 98.8 & 236 & 94.8 & \multirow[t]{2}{*}{$<0.001$} \\
\hline MAP & 78 & 1.2 & 13 & 5.2 & \\
\hline \multicolumn{6}{|c|}{ Private health insurance } \\
\hline Yes & 5628 & 85.8 & 199 & 79.9 & \multirow[t]{2}{*}{0.009} \\
\hline No & 930 & 14.2 & 50 & 20.1 & \\
\hline \multicolumn{6}{|c|}{ Monthly household income, USD ${ }^{a}$} \\
\hline$<2500$ & 1059 & 16.2 & 56 & 22.5 & \multirow[t]{3}{*}{0.013} \\
\hline 2500-4499 & 3199 & 48.8 & 103 & 41.4 & \\
\hline$\geq 4500$ & 2300 & 35.1 & 90 & 36.1 & \\
\hline \multicolumn{6}{|c|}{ Time since most recent Pap smear test, years } \\
\hline$<1$ & 1985 & 30.3 & 77 & 30.9 & \multirow[t]{4}{*}{0.003} \\
\hline $1-2$ & 2260 & 34.5 & 77 & 30.9 & \\
\hline$>2$ & 628 & 9.6 & 41 & 16.5 & \\
\hline Never & 1685 & 25.7 & 54 & 21.7 & \\
\hline
\end{tabular}

NHI National Health Insurance, MAP Medical Aid Program

${ }^{\mathrm{a}} 1 \mathrm{USD}=1000 \mathrm{KWN}$

2 years, despite the consensus of recommendations that women discontinue Pap smear examinations if they undergo a total hysterectomy following a diagnosis with a benign disease. Among women who did not undergo hysterectomies, $64.7 \%$ reported that they had undergone cervical cancer screening within the same period.

There are some possible explanations for the unnecessary screening of women who underwent hysterectomies. First, such women are unlikely to recognize that cervical cancer screening is no longer necessary. Second, it is possible that doctors may not be aware that women without cervices no longer require cervical cancer screening. Third, even if the doctors are aware of the recommendations, it is possible that they did not advise their patients of the same. In the absence of a shared medical record system, it is difficult for doctors to determine whether a patient underwent a total or subtotal hysterectomy; hence, a physician may not wish to encourage a patient to forgo cancer screening without knowledge of whether the patient is at a high risk for cervical cancer. Fourth, various medical environments and systems may produce unnecessary screenings. For example, 1 - some screening examination systems are not under the coordination of the primary care physician in the primary care setting, 2- the national medical checkup system is followed automatically without consulting a physician beforehand, and 3- the fee-for-service system drives physicians to see more patients for shorter 
Table 3 Cervical cancer screening rates according to hysterectomy status and sociodemographic variables

\begin{tabular}{|c|c|c|c|c|c|c|c|c|}
\hline \multirow[t]{2}{*}{ Characteristics } & \multicolumn{2}{|c|}{ Reporting no hysterectomy } & \multicolumn{2}{|c|}{ Reporting hysterectomy } & \multicolumn{4}{|c|}{ Reporting hysterectomy vs. reporting no hysterectomy } \\
\hline & Screening rate & $(95 \% \mathrm{Cl})$ & Screening rate & $(95 \% \mathrm{Cl})$ & COR & $(95 \% \mathrm{Cl})$ & $\mathrm{aOR}$ & $(95 \% \mathrm{Cl})$ \\
\hline Overall & 64.7 & $(64.1-65.3)$ & 61.8 & $(58.8-64.9)$ & 0.87 & $(0.67-1.13)$ & 0.75 & $(0.57-0.98)$ \\
\hline \multicolumn{9}{|l|}{ Age, years } \\
\hline $30-44$ & 57.1 & $(56.1-58.1)$ & 77.8 & $(68.0-87.6)$ & 2.70 & $(0.88-8.22)$ & 2.36 & $(0.75-7.44)$ \\
\hline $45-64$ & 70.1 & $(69.3-70.9)$ & 64.7 & $(61.0-68.4)$ & 0.77 & $(0.56-1.07)$ & 0.75 & $(0.54-1.05)$ \\
\hline $65-74$ & 64.9 & $(63.2-66.7)$ & 50.0 & $(43.8-56.3)$ & 0.55 & $(0.33-0.93)$ & 0.53 & $(0.31-0.91)$ \\
\hline \multicolumn{9}{|l|}{ Residence area } \\
\hline Metropolitan & 65.8 & $(64.9-66.7)$ & 58.2 & $(53.7-62.7)$ & 0.72 & $(0.50-1.04)$ & 0.59 & $(0.40-0.88)$ \\
\hline Urban & 64.1 & $(63.3-65.0)$ & 66.4 & $(61.9-70.8)$ & 1.07 & $(0.72-1.60)$ & 0.97 & $(0.65-1.46)$ \\
\hline Rural & 62.0 & $(59.9-64.2)$ & 57.1 & $(43.9-70.4)$ & 0.83 & $(0.28-2.44)$ & 0.75 & $(0.24-2.31)$ \\
\hline \multicolumn{9}{|l|}{ Education, years } \\
\hline$\leq 11$ & 67.2 & $(65.7-68.8)$ & 64.7 & $(58.9-70.5)$ & 0.82 & $(0.49-1.39)$ & 0.92 & $(0.54-1.56)$ \\
\hline$\geq 12$ & 64.3 & $(63.7-65.0)$ & 60.8 & $(57.1-64.4)$ & 0.87 & $(0.64-1.18)$ & 0.69 & $(0.50-0.95)$ \\
\hline \multicolumn{9}{|c|}{ Family history of cancer } \\
\hline Yes & 67.1 & $(65.5-68.7)$ & 75.0 & $(70.2-79.8)$ & 1.47 & $(0.87-2.49)$ & 1.42 & $(0.82-2.44)$ \\
\hline No & 64.4 & $(63.7-65.0)$ & 55.6 & $(51.8-59.4)$ & 0.69 & $(0.50-0.94)$ & 0.59 & $(0.43-0.81)$ \\
\hline \multicolumn{9}{|c|}{ Health insurance status } \\
\hline $\mathrm{NHI}$ & 64.8 & $(64.2-65.4)$ & 62.7 & $(59.6-65.9)$ & 0.90 & $(0.69-1.18)$ & 0.75 & $(0.57-0.99)$ \\
\hline MAP & 57.7 & $(52.1-63.3)$ & 46.2 & $(32.3-60.0)$ & 0.64 & $(0.19-2.15)$ & 0.86 & $(0.19-3.90)$ \\
\hline \multicolumn{9}{|c|}{ Private health insurance } \\
\hline Yes & 66.0 & $(65.4-66.7)$ & 62.8 & $(59.4-66.2)$ & 0.88 & $(0.65-1.17)$ & 0.70 & $(0.52-0.95)$ \\
\hline No & 56.9 & $(55.3-58.5)$ & 58.0 & $(51.0-65.0)$ & 0.96 & $(0.54-1.73)$ & 1.01 & $(0.55-1.85)$ \\
\hline \multicolumn{9}{|c|}{ Monthly household income, USD } \\
\hline$<2500$ & 65.3 & $(63.9-66.8)$ & 48.2 & $(41.5-54.9)$ & 0.48 & $(0.28-0.82)$ & 0.49 & $(0.28-0.86)$ \\
\hline $2500-4499$ & 61.9 & $(61.0-62.7)$ & 63.1 & $(58.4-67.9)$ & 1.03 & $(0.69-1.55)$ & 0.86 & $(0.57-1.31)$ \\
\hline$\geq 4500$ & 68.4 & $(67.5-69.4)$ & 68.9 & $(64.0-73.8)$ & 1.05 & $(0.66-1.65)$ & 0.87 & $(0.54-1.41)$ \\
\hline
\end{tabular}

$\mathrm{Cl}$ confidence intervals, $c O R$ crude odds ratio, $a O R$ adjusted odds ratio, Adjustments were made for age, residence area, education, family history of cancer, health insurance status, private health insurance, and monthly household income, NHI National Health Insurance, MAP Medical Aid Program

${ }^{\mathrm{a}} 1 \mathrm{USD}=1000 \mathrm{KWN}$

durations; all these factors may help explain our findings.

Despite several decades having passed since the publication of recommendations for women who underwent hysterectomy in the United States, cervical cancer screening rates in that country remain high. In 1992, 68.5\% of women with a history of hysterectomy reported having had cervical cancer screening within the preceding three years. In 2002, 69.1\% had undergone screening during the previous three years, which was not significantly different than the 1992 rate despite the 1996 USPTF recommendation that routine cervical cancer screening is unnecessary for women who have undergone a complete hysterectomy for benign disease [21]. In 2010, 64.8\% of women in the United States with a history of hysterectomy reported having undergone cervical cancer screening; among women with no hysterectomies, $80.7 \%$ reported having undergone screening. This showed that unnecessary cervical cancer screening persists in women who have undergone hysterectomies [17].

According to the multivariable analyses that compared Pap smear screening rates between women with vs. without hysterectomies, women with hysterectomies who were residents of metropolitan areas, those with higher educational levels, those with higher incomes, and those with National Health Insurance underwent fewer Pap smear screening tests than those without hysterectomies. This result implies that subjects who are more sensitive and literate to health information, or are more amenable to healthcare guidance, actually follow recommendations and undergo less screening. Poor literacy is known to be linked to low socio-economic circumstances to some extent [22], and previous studies have shown that women with higher levels of education are less likely to undergo screening tests after being informed that such cancer screening is unnecessary [23]. 
However, in younger women (30-44 years) or women with a family history of cancer, those who had undergone hysterectomies showed higher cervical cancer screening rates than those who had not. In a recent Korean study, women who strongly believed that cancer screening facilitates early detection were less likely to change their intentions to undergo screening for thyroid cancer after receiving information about overdiagnosis [23]. In another study on breast cancer screening in the United Kingdom, women eligible for cost-free breast cancer screening under a nationwide screening program were less likely to defer continued screening after receiving information on overdiagnosis than women not eligible for such screening because of younger age [24]. Such individuals are likely to have greater interests and concerns about cancer. Therefore, while they actively seek health information on one hand but ignore guidelines regarding the non-necessity of screening on the other, as such recommendations may increase their anxiety or cause mental discomfort [25]. Once formed, ideas on healthcare are not easily changed because of a single piece of additional information. Given the experiences in the United States, it is important to understand selectivity in receiving information and provide accurate information repetitively.

Our study has some limitations. First, data were collected using self-reported questionnaires; hence, recall bias may exist and the estimates should be interpreted with caution. The validity of self-reported cancer screening may be over-reported [26], especially in women [27]. Nevertheless, self-reported screening behavior generally is in good agreement with medical records [28, 29], and many publications rely on this. Second, over- or underestimation may occur when determining the unnecessary cervical cancer screening rates owing to the lack of detail on the types or reasons for hysterectomies in the KNCSS data. Especially, we could not distinguish between total and subtotal (supracervical) hysterectomies, which remove only the uterus and leave the cervix intact, among women who answered that they had undergone a hysterectomy. Women with subtotal hysterectomies would still be recommended for screening, and this could, to some extent, affect our results, i.e. high screening rates among hysterectomized women. However, Korea has reportedly shown a relatively low proportion of cervical conservation, of $6.8 \%$ among overall hysterectomies in 2008 [10], which means that the impact will be limited. In future studies, it will be necessary to determine cervical cancer screening rates according to specific hysterectomy types (total hysterectomy or subtotal hysterectomy) as well as the reasons for them (i.e., benign or malignant conditions). Third, we could not determine causal associations between cervical cancer screening and hysterectomy status and other associated factors because the KNCSS uses a cross-sectional design. Fourth, the percent who reported having a hysterectomy - 3.7\%- was likely underestimated although all participants responded to the question of hysterectomy history. It is possible that this has affected the outcome of our study. Despite such limitations, our study ought to be worthwhile as it is the first to determine the rate of unnecessary Pap smear screening among women with hysterectomies in Korea using nationwide representative, population-based data.

\section{Conclusions}

In conclusion, our investigation revealed that a considerable number of women without cervices in Korea undergo unnecessary cervical cancer screening contrary to evidence-based clinical recommendations. Performing unnecessary screening can cause various side effects including psychosocial stress, wasting of limited healthcare resources, and ultimately increased healthcare expenses. It is necessary to identify the exact underlying causes for pursuing unnecessary screening, and systematic efforts are required to reduce such screening for women who have undergone total hysterectomies.

\section{Additional file}

Additional file 1: Questionnaire hysterectomy and Pap screening. (DOCX $18 \mathrm{~kb}$ )

\section{Abbreviations}

CIN: Cervical intraepithelial neoplasia; KNCCS: Korean National Cancer Screening Survey; NCSP: National Cancer Screening Program; USPSTF: United States Preventive Services Task Force

\section{Funding}

This study was supported by a Grant-in-Aid for Cancer Research and Control from the National Cancer Center of Korea (\# 1610402). Funding bodies have no role in the study design, study setting, analysis, or writing of the manuscript.

\section{Availability of data and materials}

The dataset supporting the conclusions of this article is available from the corresponding author on reasonable request.

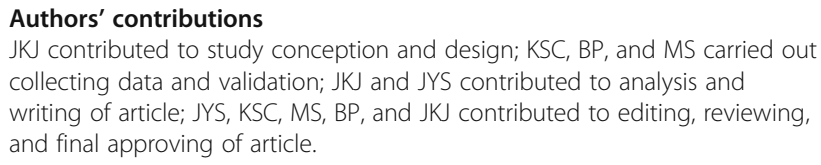

Ethics approval and consent to participate

This study was approved by the Institutional Review Board of the National Cancer Center, Korea (NCCNCS-08-129). Written informed consent was obtained from all individual participants in the study.

Consent for publication

Not applicable.

Competing interests

The authors declare that they have no competing interests. 


\section{Publisher's Note}

Springer Nature remains neutral with regard to jurisdictional claims in published maps and institutional affiliations.

\section{Author details}

${ }^{1}$ Department of Preventive Medicine, School of Medicine, Kyungpook National University, Daegu 41944, Republic of Korea. ${ }^{2}$ Graduate School of Cancer Science and Policy, National Cancer Center, 323 IIsan-ro, Ilsandong-gu, Goyang 10408, Republic of Korea. ${ }^{3}$ National Cancer Control Institute, National Cancer Center, 323 IIsan-ro, Ilsandong-gu, Goyang 10408 Republic of Korea. ${ }^{4}$ Department of Medicine, College of Medicine, Hanyang University, Seoul 04763, Republic of Korea.

Received: 16 April 2018 Accepted: 2 August 2018

Published online: 11 August 2018

\section{References}

1. Ferlay J, Soerjomataram I, Dikshit R, Eser S, Mathers C, Rebelo M, et al. Cancer incidence and mortality worldwide: sources, methods and major patterns in GLOBOCAN 2012. Int J Cancer. 2015;136:E359-86.

2. Lee NC, Wong FL, Jamison PM, Jones SF, Galaska L, Brady KT, et al. Implementation of the National Breast and Cervical cancer Early Detection Program: the beginning. Cancer. 2014;120(suppl 16):2540-8.

3. DeGroff A, Carter A, Kenney K, Myles Z, Melillo S, Royalty J, et al. Using evidence-based interventions to improve cancer screening in the National Breast and Cervical Cancer Early Detection Program. J Public Health Manag Pract. 2016:22:442-9

4. Bray F, Loos AH, McCarron P, Weiderpass E, Arbyn M, Møller H, et al. Trends in cervical squamous cell carcinoma incidence in 13 European countries: changing risk and the effects of screening. Cancer Epidemiol Biomark Prev. 2005:14:677-86.

5. Ward KK, Shah NR, Saenz CC, McHale MT, Alvarez EA, Plaxe SC. Changing demographics of cervical cancer in the United States (1973-2008). Gynecol Oncol. 2012;126:330-3

6. Moyer VA, U.S. Preventive Services Task Force. Screening for cervical cancer: U.S. Preventive Services Task Force recommendation statement. Ann Intern Med. 2012;156:880-91.

7. Suh $M$, Choi KS, Park B, Lee $Y Y$, Jun JK, Lee DH, et al. Trends in cancer screening rates among Korean men and women: results of the Korean National Cancer Screening Survey, 2004-2013. Cancer Res Treat. 2016:48:1-10.

8. Jung KW, Won YJ, Oh CM, Kong HJ, Lee DH, Lee KH. Cancer statistics in Korea: incidence, mortality, survival, and prevalence in 2014. Cancer Res Treat. 2017:49:292-305.

9. Merrill RM. Hysterectomy surveillance in the United States, 1997 through 2005. Med Sci Monit. 2008;14:CR24-31.

10. Lee EJ, Park HM. Trends in laparoscopic surgery for hysterectomy in Korea between 2007 and 2009. J Obstet Gynaecol Res. 2014;40:1695-9.

11. Keshavarz H, Hillis SD, Kieke BA, Marchbanks PA. Hysterectomy surveillance-United States, 1994-1999. MMWR Surveill Summ. 2002;51:1-8.

12. Korea National Health Insurance Service. Main surgery statistical yearbook 2015. Wonju: National Health Insurance Service; 2016.

13. US Preventive Services Task Force. Guide to Clinical Preventive Services. 2nd ed. Baltimore: Williams \& Wilkins; 1996. p. 105-18.

14. Partridge EE, Abu-Rustum NR, Campos SM, Fahey PJ, Farmer M, Garcia RL, et al. Cervical cancer screening: clinical practice guidelines in oncology. J Natl Compr Cancer Netw. 2010;8:1358-86.

15. Saslow D, Solomon D, Lawson HW, Killackey M, Kulasingam SL, Cain J, et al, American Cancer Society, American Society for Colposcopy and Cervical Pathology, and American Society for Clinical Pathology screening guidelines for the prevention and early detection of cervical cancer. CA Cancer J Clin. 2012;62:147-72

16. Min KJ, Lee YJ, Suh M, Yoo CW, Lim MC, Choi J, et al. The Korean guideline for cervical cancer screening. J Gynecol Oncol. 2015:26:232-9.

17. Kepka D, Breen N, King JB, Benard VB, Saraiya M. Overuse of papanicolaou testing among older women and among women without a cervix. JAMA Intern Med. 2014:174:293-6.

18. Selvin E, Brett KM. Breast and cervical cancer screening: sociodemographic predictors among White, Black, and Hispanic women. Am J Public Health. 2003;93:618-23.

19. Hahm Ml, Chen HF, Miller T, O'Neill L, Lee HY. Why do some people choose opportunistic rather than organized cancer screening? The Korean National
Health and Nutrition Examination Survey (KNHANES) 2010-2012. Cancer Res Treat. 2017:49:727-38.

20. US Preventive Services Task Force. Screening for cervical cancer: recommendations and rationale. Am J Nurs. 2003:103:101-9.

21. Sirovich BE, Welch HG. Cervical cancer screening among women without a cervix. JAMA. 2004;291:2990-3.

22. Nutbeam D. The evolving concept of health literacy. Soc Sci Med. 2008;67:2072-8.

23. Lee S, Lee YY, Yoon HJ, Choi E, Suh M, Park B, et al. Responses to overdiagnosis in thyroid cancer screening among Korean women. Cancer Res Treat. 2016;48:883-91.

24. Waller J, Whitaker KL, Winstanley K, Power E, Wardle J. A survey study of women's responses to information about overdiagnosis in breast cancer screening in Britain. Br J Cancer. 2014:111:1831-5.

25. Case DO, Andrews JE, Johnson JD, Allard SL. Avoiding versus seeking: the relationship of information seeking to avoidance, blunting, coping, dissonance, and related concepts. J Med Libr Assoc. 2005;93:353-62.

26. Rauscher GH, Johnson TP, Cho Yl, Walk JA. Accuracy of self-reported cancerscreening histories: a meta-analysis. Cancer Epidemiol Biomark Prev. 2008;17:748-57.

27. Howard M, Agarwal G, Lytwyn A. Accuracy of self-reports of Pap and mammography screening compared to medical record: a meta-analysis. Cancer Causes Control. 2009;20:1-13.

28. Caplan LS, McQueen DV, Qualters JR, Leff M, Garrett C, Calonge N. Validity of women's self-reports of cancer screening test utilization in a managed care population. Cancer Epidemiol Biomark Prev. 2003:12:1182-7.

29. Jones RM, Mongin SJ, Lazovich D, Church TR, Yeazel MW. Validity of four self-reported colorectal cancer screening modalities in a general population: differences over time and by intervention assignment. Cancer Epidemiol Biomark Prev. 2008;17:777-84.

30. Lee JK, Hong JH, Kang S, Kim DY, Kim BG, Kim SH, et al. Practice guidelines for the early detection of cervical cancer in Korea: Korean Society of Gynecologic Oncology and the Korean Society for Cytopathology 2012 edition. J Gynecol Oncol. 2013;24:186-203.

Ready to submit your research? Choose BMC and benefit from

- fast, convenient online submission

- thorough peer review by experienced researchers in your field

- rapid publication on acceptance

- support for research data, including large and complex data types

- gold Open Access which fosters wider collaboration and increased citations

- maximum visibility for your research: over $100 \mathrm{M}$ website views per year

At BMC, research is always in progress.

Learn more biomedcentral.com/submissions 